\title{
Auf welcher Seite des Baumes können Reflexionsfolien die Fruchtfarbe stärker verbessern?
}

\author{
Patrick Hess $^{1} \cdot$ Michael Blanke $^{1}$
}

Eingegangen: 20. Juni 2019 / Angenommen: 1. April 2021 / Online publiziert: 9. Juni 2021

(c) Der/die Autor(en) 2021

\section{Zusammenfassung}

Im Rahmen einer Masterarbeit sollte untersucht werden, wie weit und von welcher Seite des Baumes bzw. Baumreihe Reflexionsfolien aus Kunststoff (in der Fahrgasse) und recyceltem Aluminium (direkt unter den Bäumen) zur Verbesserung der Fruchtfarbe im Herbst beitragen.

Aus früheren Arbeiten war bekannt, dass die Lichtstrahlung durch Gewebefolien weit in die Baumkrone reflektiert wird, aber nicht, ob diese Lichtstärke ausreicht, die Äpfel in verschiedenen Regionen der Baumkrone besser zu färben und ob Aluminiumfolie die gleiche Wirkung zeigt.

Daher wurden 90 neun Jahre alte Apfelbäume der Sorte 'Braeburn, Hillwell' auf M 9 unter grauem Hagelnetz auf dem Campus Klein-Altendorf der Universität Bonn im September 2018 - einem Jahr mit guter Fruchtausfärbung - untersucht. Die Apfelbäume waren als schlanke Spindel erzogen und die Reihen zur Optimierung der Lichtnutzung in N-S-Ausrichtung gepflanzt. Die Gewebefolie Lumilys mit 2,6 m Breite wurde in den Fahrgassen und eine Folie aus $80 \%$ recyceltem Aluminium $2 \times 60 \mathrm{~cm}$ breit direkt unter den Bäumen ca. 6 Wochen vor dem geschätzten Erntetermin ausgelegt. Als chemische Variante wurden Bäume mit dem Biostimulanz Stimplex ${ }^{\mathrm{TM}}$ mit $2 \times 4$ 1/ha nach Herstellerempfehlung behandelt; Bäume der gleichen Reihen ohne Behandlung dienten als Kontrolle. Ab 6 Wochen vor der Ernte wurden 3600 Farbwinkelmessungen an den gleichen 180 Apfelfrüchten am Baum durchgeführt mit folgenden Ergebnissen

1) Die schlechtere Rotfärbung der Apfelfrüchte auf der Ostseite (Kontrolle ca. $69^{\circ}$ hue) als auf der Westseite (ca. $51^{\circ}$ hue) kann eine Farbverbesserung auf der Ostseite des Baumes durch Folien, Chemikalien oder andere Mittel erfordern.

2) Auf dieser schlecht belichteten Ostseite zeigten die Apfelfrüchte im unteren Kronenbereich unter $1 m$ Kronenhöhe die schlechteste Rotfärbung - mit 66, $4^{\circ}$ hue bei Stimplex, 69, $1^{\circ}$ hue in der Kontrolle und 70,4 hue bei Folienwechsel.

3) Auf dieser schlecht belichteten Ostseite unter $1 \mathrm{~m}$ Kronenhöhe zeigten die Reflexionsfolien die stärkste Wirkung - mit guter Rotfärbung der Apfelfrüchte direkt über der Aluminiumfolie (53,3hue) und sowohl über der Gewebefolie Lumilys in jeder $\left(59,6^{\circ}\right.$ hue $)$ als auch jeder 2 . Reihe $\left(57^{\circ}\right.$ hue $)$.

4) Auf der gut belichteten Westseite im Kronenbereich über $1 \mathrm{~m}$ zeigten die Reflexionsfolien dagegen eine geringe Wirkung; nur in diesem Bereich verbesserten weder die Aluminium- noch die Textilfolie Lumilys die Rotfärbung.

5) Das Auslegen der Gewebefolie Lumilys in jeder 2. Reihe bzw. Fahrgasse erzielte eine gleich gute Verbesserung der Rotfärbung wie das Auslegen in jeder Reihe und die (recycelte) Aluminiumfolie direkt unter den Bäumen.

Generell wirkten die Reflexionsfolien - unabhängig von Material und Auslegen - auf die Fruchtausfärbung in diesem Jahr 2018 am besten an den stärker beschatteten Stellen in der Baumkrone.

Schlüsselwörter Apfel (Malus domestica Borkh) · Anthocyansynthese · Biostimulanzien · Fruchtfarbe · Lichtreflexion · Mulchfolie · PAL

Michael Blanke

mmblanke@uni-bonn.de

1 INRES-Gartenbauwissenschaft, Universität Bonn, Auf dem

Hügel 6, 53121 Bonn, Deutschland 


\section{On Which Side of the Tree Are Reflective Mulches More Efficient to Improve Fruit Colour?}

\section{Abstract}

The objective of the present study was to investigate how far and from which side reflective mulches effectively reflect light into the tree crown to improve the colour of apple fruit under hail net in the autumn when light is limiting at $50^{\circ} \mathrm{N}$ latitude. Therefore, polypropylene mulch (Lumilys ${ }^{\mathrm{TM}}$ ) was spread $2.6 \mathrm{~m}$ wide in the alleyways and (recycled) aluminum foil in the herbicide strip under the trees $(0.6 \mathrm{~m}$ either side of the tree) six weeks prior to anticipated harvest.

Previous work had shown the light penetration and distribution within the tree canopy using polypropylene mulch (Lumilys ${ }^{\mathrm{TM}}$ ) in the alleyways, but without showing the effect on fruit colouration and without using other mulches or strategies.

Hence, ninety 9-year-old cv. 'Braeburn, Hillwell' apple trees on M 9 rootstock at CKA Klein-Altendorf, University of Bonn were employed for the experiment in 2018, a year with generally good fruit colouration. Trees were trained to slender spindles and were planted in N-S rows to optimize light utilization. A bio stimulant (Stimplex ${ }^{\mathrm{TM})}$ was included as a chemical colour enhancer, while untreated trees of the same rows served as control.

180 apple fruits were marked and repetitively assessed four-times prior to harvest resulting in 3600 colour measurements on attached fruit with the following results

1) Fruit on the East side of the tree developed less red colouration $\left(69^{\circ}\right.$ hue) than those on the West side, identifying the need for colour improvement measures such as reflective mulches, chemicals or other on the East side

2) Low hanging fruit under $1 \mathrm{~m}$ height in the tree canopy developed insufficient colour $\left(70.4^{\circ}\right.$ hue), which was improved by Stimplex to $66.4^{\circ}$ hue with a one week earlier harvest

3) In this otherwise shaded area, reflective mulches showed the greatest effect: Apple fruit reached good red colouration with polypropylene (Lumilys $\left.{ }^{\mathrm{TM}}\right)$ in the alleyways of every row $\left(59.6^{\circ}\right.$ hue), every other row $\left(57^{\circ}\right.$ hue) or with aluminum foil directly under the tree $\left(53.3^{\circ} \mathrm{hue}\right)$, where lower hue values represent better red colour

4) By contrast, the positive effect on colouration of the reflective mulches appeared less pronounced for fruit in the lightexposed Westside of the tree canopy, irrespective of their material and application (polypropylene in the alleyways or aluminum directly under the trees).

5) Spreading the reflective mulch Lumilys ${ }^{\mathrm{TM}}$ every second row viz alleyway improved fruit colouration, to the same extent, as spreading the same material in the traditional way in every row viz alleyway or spreading (recycled) aluminum foil directly under the trees.

Regardless of the material and spreading approach, the reflective mulches showed the best effect on fruit colouring in the more shaded parts of the tree canopy.

Keywords Apple (Malus domestica Borkh) - Anthocyanin • Biostimulant · Climate change · Consumer preference · Extenday $\cdot$ Fruit colour $\cdot$ Light reflection $\cdot$ Mulch film $\cdot$ PAL $\cdot$ Sustainability

\section{Einleitung}

In den nördlichen Breiten ab ca. $50^{\circ} \mathrm{N}$ ist Licht der begrenzende Faktor für die Photosynthese der Pflanzen und die Färbung der Früchte (Blanke und Kunz 2009). Die Kombination aus Anbau unter Hagelnetz, sinkendem Sonnenstandswinkel, abnehmender Lichtintensität, kürzerer Tageslänge (Photoperiode) und warmen Nächten im Herbst erschwert die Rotfärbung bei zweifarbigen Äpfeln (Funke und Blanke 2021). Besonders betroffen sind die Standardsorten wie 'Elstar, Elshof', 'Royal Gala' und 'Gala, Mondial' (Blanke 2015) und 'Pinova' (Bosančić et al. 2018). Die Rotfärbung der Schale wird durch die Anthocyansynthese bewirkt (Treutter 2010), die durch die Gene MdMYB1, MdMYB10 und bHLH3 (basic Helix Loop Helix) reguliert bzw. gefördert wird (Wu et al. 2012) sowie mittels des Proteins UVR8 positiv auf UV-Strahlen reagiert (Chris- tie et al. 2012). Ein hoher Anteil an roter Farbe wird von den Verbrauchern bevorzugt, weil dieser mit einem besseren Geschmack und höherer Qualität (Bosančić et al. 2018) sowie reifen (Hamadziripi et al. 2014) und süßen Früchten (Steyn et al. 2009) assoziiert wird. In der Praxis werden Anlieferungen bzw. Paletten am Großmarkt oft abgelehnt oder preislich herabgestuft, wenn sie neben gut gefärbten Früchten z.B. von 'Braeburn, Maririred' schlecht gefärbte Früchte von 'Braeburn, Hillwell' enthalten. Bisher war bekannt, dass gewebte Reflexionsfolien aus Polypropylen wie Extenday oder Lumilys die Fruchtfärbung verbessern und die Fruchtfestigkeit erhalten (z. B. Schuhknecht et al. 2018) und dass die reflektierten Lichtstrahlen relativ hoch in die Baumkrone eindringen (Weber et al. 2019). Unbekannt bzw. unerforscht war, ob ihre Wirkung ausreicht, die Fruchtfarbe in verschiedenen Teilen der Baumkrone zu verbessern und auf welcher Baumseite bzw. -reihe (Ost oder West) die 
Abb. 1 Verschiedene Ansätze zur Verwendung von a gewebter Kunststofffolie Lumilys (links) bzw. b (recycelter) Aluminiumfolie direkt unter den Bäumen nach 6-wöchiger Exposition am Ende des Versuchs kurz vor der Ernte

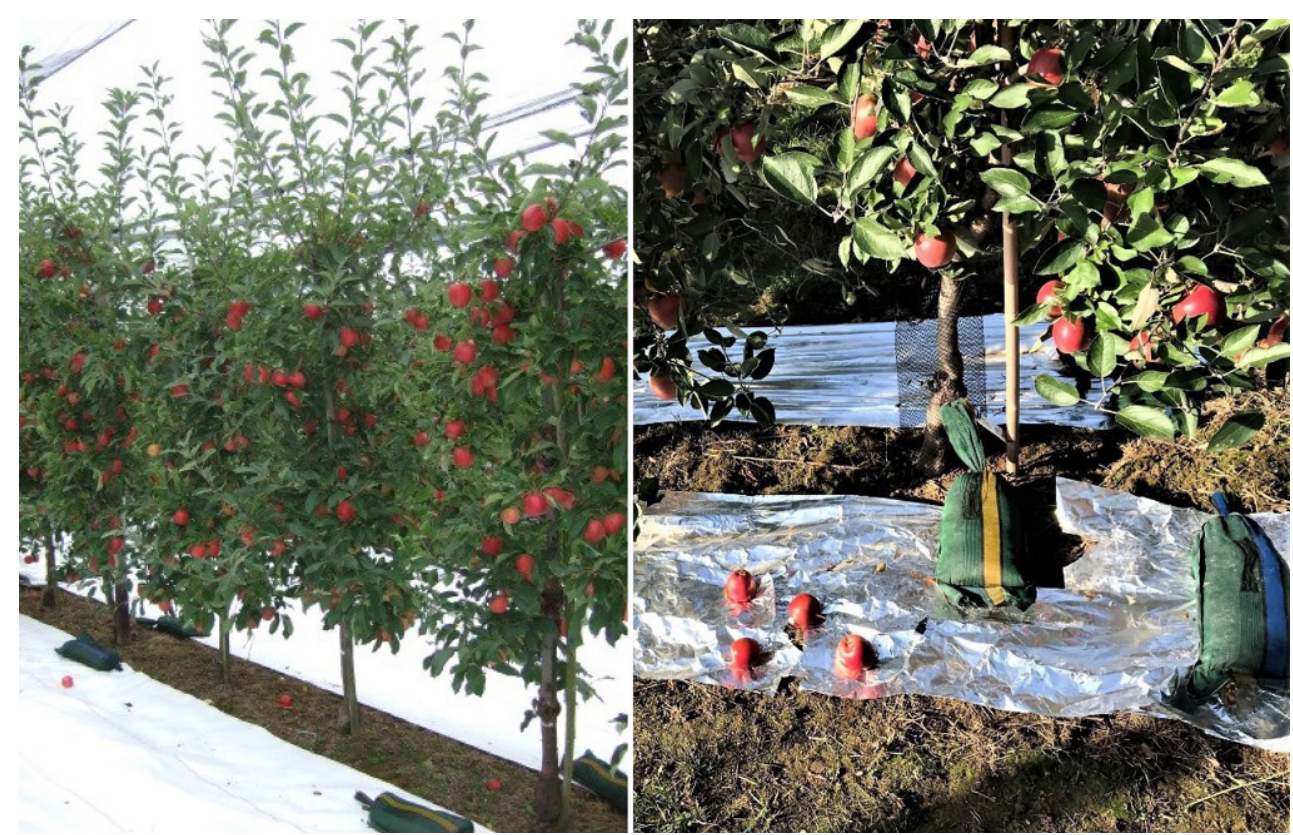

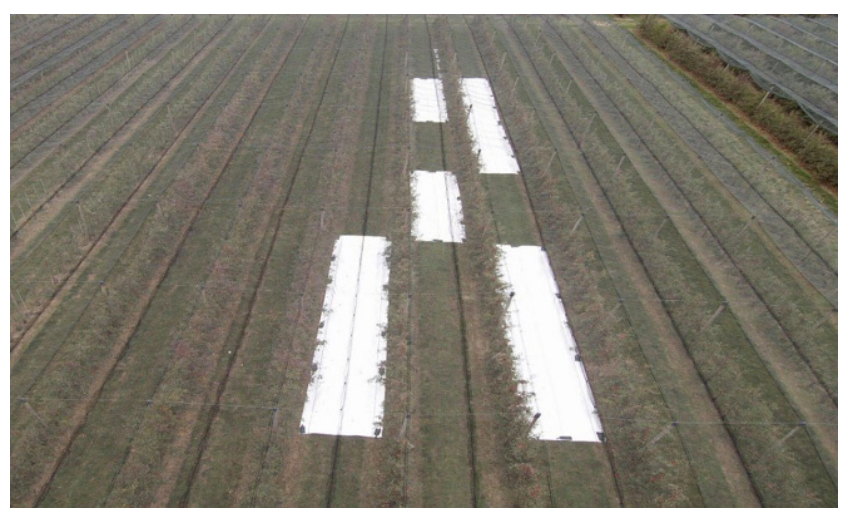

Abb. 2 Luftaufnahme von A. Kunz des Versuchs in Klein-Altendorf mit den Kunststofffolien im Vordergrund und der Aluminiumfolie unter den Bäumen im Hintergrund im Jahre 2018. Bäume ohne Folie dienten der chemischen Behandlung oder als Kontrolle

Reflexionsfolien effektiver sind. Bisher kamen dazu u.a. nassfeste Papiere (Meinhold et al. 2011) und weiße Gewebefolien (Schuhknecht et al. 2018; Weber et al. 2019) zum Einsatz, da bislang keine recycelte Aluminiumfolie zur Verfügung stand. Frühe Arbeiten (Meinhold et al. 2011) hatten bereits gezeigt, dass fabrikneue Aluminiumfolien - im Gegensatz zu Gewebefolien aus Kunststoff-Licht gerichtet, d. h. vornehmlich direkt nach oben reflektieren, so dass sie am effektivsten direkt unter den Bäumen wirkt.

Daher sollte im Rahmen dieser Arbeit die Wirkung einer weißen gewebten Polypropylen-Reflexionsfolie (Lumilys) und - zum ersten Mal in Klein-Altendorf - einer aus $80 \%$ recycelten glatten Aluminium bestehenden Folie auf die Apfelfrüchte - differenziert nach West- und Ostseite in verschiedenen Baumhöhen bei der späten Sorte 'Braeburn, Hillwell` aufgezeigt werden.

\section{Material und Methoden}

\section{Versuchsstandort, Apfelbäume, Reflexionsfolien und Biostimulanz}

Das Versuchsgelände des CKA Klein-Altendorf $\left(6^{\circ} 59^{\prime} \mathrm{E}\right.$, $50^{\circ} 37^{\prime} \mathrm{N}, 180 \mathrm{~m}$ ü $\mathrm{NN}$ ) der Universität Bonn ist durch nordwestliche Atlantikwinde und ein gleichbleibendes gemäßigtes Klima geprägt. Die Jahresmitteltemperatur beträgt $9,8^{\circ} \mathrm{C}$ mit ca. $600 \mathrm{~mm}$ Niederschlag (Blanke und Kunz 2009). Der Versuch wurde mit der Apfelsorte 'Braeburn, Hillwell' auf M 9 unter grauem Hagelnetz durchgeführt. Die Reihen waren in Nord-Süd Richtung gepflanzt. Die Spindelbäume waren 9 Jahre alt mit einer Pflanzweite von $3,5 \mathrm{~m} \times 1 \mathrm{~m}$ Baumabstand.

Im Versuch wurden die gewebte Kunststofffolie Lumilys (Beaulieu, Belgien, Abb. 1a) (Lumilys 2018), eine Aluminiumfolie der Marke Profissimo (Abb. 1b) und das Biostimulanz Stimplex (Acadian Plant Health, Kanada) zur Verbesserung der Fruchtqualität und vor allem der Fruchtfarbe verwendet. Die Aluminiumfolie und Lumilys wurden am 30. August 2018 ca. 6 Wochen vor der Ernte ausgelegt (Tab. 1). Lumilys wurde auf 2,6m Breite in der Fahrgasse ausgerollt, die Aluminiumfolie wurde jeweils $60 \mathrm{~cm}$ links und rechts direkt unter den Bäumen ausgelegt (Abb. 2).

Die Reflexionsfolie Lumilys wurde in drei Varianten ausgelegt a) In jeder Reihe bzw. Fahrgasse b) alternierend in 
Tab. 1 Termine der Folienauslegung und -verschiebung, Behandlung mit den Chemikalien (Biostimulanz Stimplex) und Farbmessungen in KleinAltendorf 2018

\begin{tabular}{llll}
\hline Variante & Auslegetermin & Behandlung/bzw. Verschieben der Gewebefolie & Termine der Farbmessungen \\
\hline Kontrolle & Entfällt & Entfällt (Gras) & 14.09 .2018 \\
Recycelte Aluminiumfolie & 30.08 .2018 & (Entfällt) & 21.09 .2018 \\
Gewebefolie Lumilys & 30.08 .2018 & (Entfällt) & 28.09 .2018 \\
Wechsel Lumylis P.1 $^{\mathrm{a}}$ & 30.08 .2018 & $12.09 . ; 25.09 . ;$ & 08.10 .2018 \\
Wechsel Lumilys P.2 $^{\mathrm{a}}$ & 30.08 .2018 & 04.10 .2018 & \\
Lumilys in alternierenden Reihen & 30.08 .2018 & (Entfällt) & \\
Biostimulanz Stimplex & (Entfällt) & $14.09 .+01.10 .18$ & \\
\hline
\end{tabular}

a siehe Abb. 3

jeder 2. Reihe bzw. Fahrgasse und c) in den Parzellen 1 und 2 wurde sie mehrfach verschoben (Abb. 3).

\section{Wiederholte Farbmessungen der Früchte am Baum}

Bei den Farbmessungen der Früchte am Baum wurden 5 Äpfel in je vier Quadranten ausgewählt: a) auf der Westseite sowie b) auf der Ostseite jeweils in einer Baumhöhe von c) 1-1,5 m und d) 0,5-1 m. Diese insgesamt 180 Äpfel wurden $4 \times$ entlang des Apfeläquators d.h. alle/je $90^{\circ}$ sowie an der stärksten rot gefärbten Stelle der Fruchtunterseite markiert. An jeder dieser Markierungen wurde die Fruchtfarbe in der CIE-Lab Farbskala an 4 Terminen während der Reifephase bzw. Anthozyansynthese ab 35 Tage vor der Ernte mit einem tragbaren i1Pro-Spektrometer (Xrite, Michigan, USA) jeweils zwischen 9 und $12 \mathrm{~h}$ gemessen (Tab. 1) und die Lab-Werte nach McGuire (1992) in ${ }^{\circ}$ hueFarbwinkel umgerechnet.

\section{Statistik}

Das Experiment umfasste 90 Apfelbäume. Jede Behandlung bestand aus zehn Bäumen plus einem Trennbaum. In einem Zeitraum von 6 Wochen vor der Ernte wurden 3600 Farbwinkelmessungen an 180 Apfelfrüchten durchgeführt. Die 9 Varianten bestanden aus je 20 Früchten, wobei eine Frucht als Wiederholung galt, und $4 \times$ über die Reifezeit gemessen wurden. Die Farbwinkel wurden auf Normalverteilung und Varianzhomogenität geprüft und anschließend einer einfaktoriellen Varianzanalyse (ANOVA) unter Verwendung der statistischen Funktion von Excel bei einem Fehler von $5 \%$ unterzogen.

\section{Ergebnisse}

\section{Rotfärbung der Früchte auf der West- versus Ostseite der Baumreihe}

Beim letzten, 4. Messtermin erreichten die Früchte bei den nicht-destruktiven Farbmessungen im Feld Farbwinkel von 50-60hue (rot) (Abb. 4) - mit Ausnahme der Ostseite unter $1 \mathrm{~m}$ Höhe. Die stärkste Anthocyansynthese - gemessen als Verringerung der hue-Farbwinkel - zeigten die Früchte zwischen dem 21. und 28. September - unabhängig von ihrer Position in der Baumkrone.

Auf der Westseite der Apfelbäume waren die hue-Farbwinkel der Früchte geringer, d.h. sie waren stärker rot gefärbt als auf der Ostseite - besonders bei Früchten aus der Kontrolle mit unbedecktem Gras und auch bei Früchten mit dem Biostimulanz Stimplex ${ }^{\mathrm{TM}}$.

\section{Einfluss der Position der Früchte im Baum - Baumhöhe - auf die Rotfärbung}

Im mittleren Bereich der Baumkrone über $1 \mathrm{~m}$ erreichten die Äpfel auf beiden Seiten der Apfelbäume bzw.- reihe, Ost und West, hue-Farbwinkel von 50-60hue. Auf der Westseite reichten die hue-Farbwinkel der Äpfel sowohl unter $1 \mathrm{~m}$ als auch über $1 \mathrm{~m}$ Höhe von $50^{\circ}$ hue (Wechsel P. 1) bis zu $62^{\circ}$ hue (Wechsel P. 2).

Früchte der Kontrollbäume ohne Behandlung, mit dem Biostimulanz Stimplex oder dem Folienwechsel P. 2 zeigten die größten hue-Farbwinkel und somit die geringste Rotfärbung der Äpfel - mit Ausnahme der Westseite in einer Höhe über $1 \mathrm{~m}$. Der Folienwechsel in P. 2 zeigte auch hier mit $61,4^{\circ}$ hue den höchsten Wert und somit die schlechteste Rotfärbung der Äpfel. Im Gegensatz dazu zeigten Früchte auf der Westseite über $1 \mathrm{~m}$ - mit dem Biostimulanz Stimplex mit $50^{\circ}$ hue und der Kontrolle mit Gras mit $51^{\circ}$ hue die geringsten Werte und somit die beste Rotfärbung. Im Kronenbereich über $1 \mathrm{~m}$ auf der gut belichteten Westseite zeigten die Reflexionsfolien - trotz hoher Lichtreflexion nur noch eine geringfügige Wirkung auf die Fruchtfärbung: 
Abb. 3 Bildliche Darstellung des Verschiebens der Lumilys Versuchszeitraums Reflexionsfolie innerhalb des
Periode 1 30.08. - 12.09.18 Parzelle Parzelle 1

\begin{tabular}{c|c} 
Lumilys & Gras \\
\hline Gras & Lumilys
\end{tabular}

Periode 2 12.09. - 25.09.18 Parzelle Parzelle

\begin{tabular}{c|c} 
Gras & Lumilys \\
\hline Lumilys & Gras
\end{tabular}

2
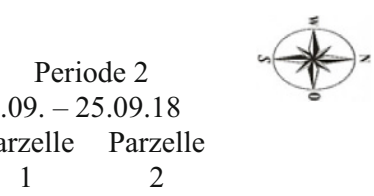

Abb. 4 Einfluss der Reflexionsfolien Lumilys ${ }^{\mathrm{TM}}$ oder Aluminiumfolie oder chemischer Behandlung (Biostimulanz Stimplex ${ }^{\mathrm{TM}}$ ) auf die Rotfärbung der Äpfel als ${ }^{\circ}$ hue-Farbwinkel im oberen bzw. unteren Teil sowie auf der Ost- und Westseite der Baumkrone im warmen September 2018 in Klein-Altendorf (mit SE (Standard Error) bei Wechsel P 2; $n=2800$ Messungen)
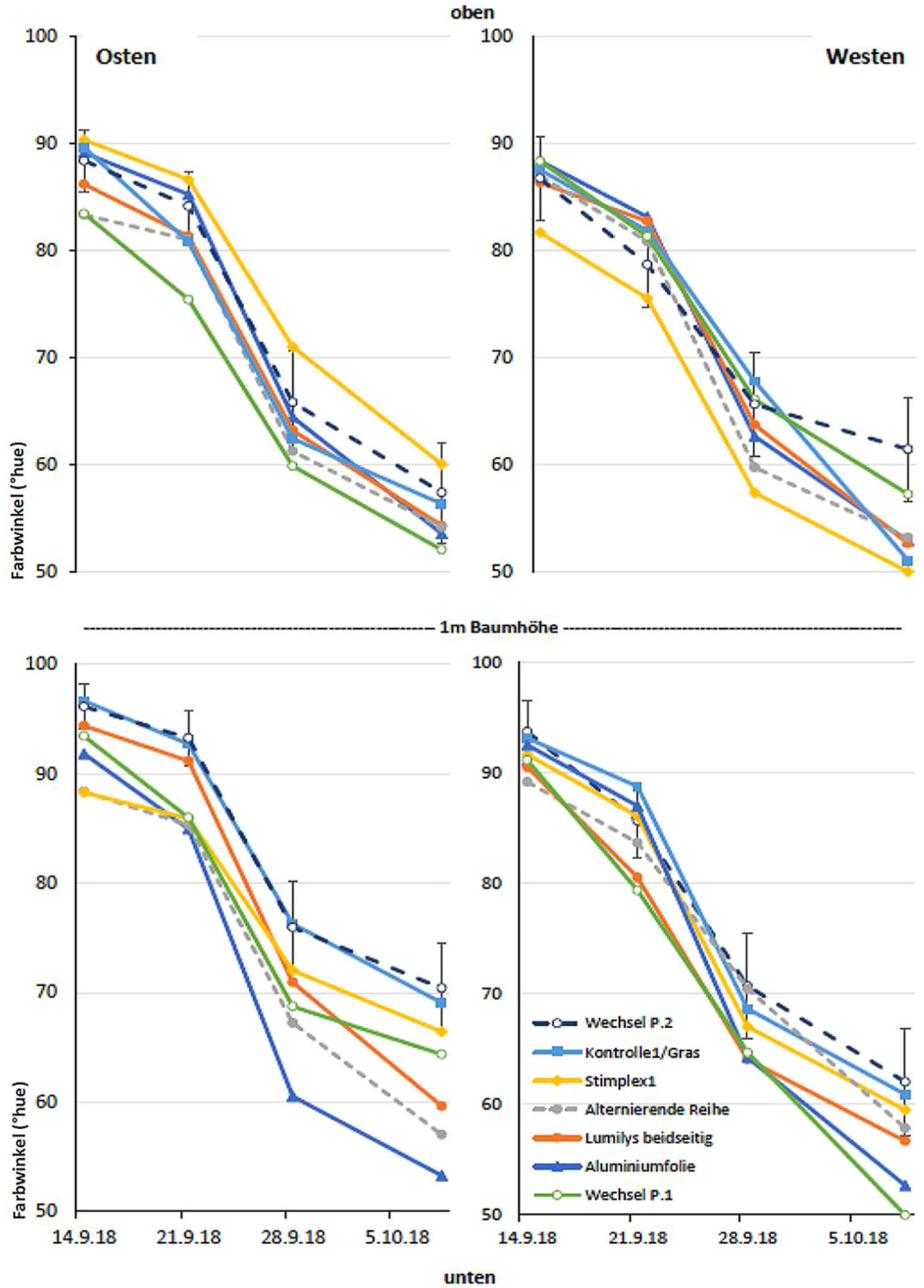
Tab. 2 Summe der UVB-Strahlung und photosynthetisch aktive Strahlung sowie der Mittelwert pro Tag für die Messzeiträume vom 30.08.-11.09.2018, 12.09.-24.09.2018, 25.09-03.10.2018 und 04.10-19.10.2018 in Klein-Altendorf

\begin{tabular}{llcccc}
\hline Periode & Messzeitraum & $\begin{array}{l}\text { PAR Summe } \\
\left(\mathrm{mmol} / \mathrm{m}^{2} \mathrm{~s}\right)\end{array}$ & $\begin{array}{l}\text { UVB Summe } \\
\left(\mathrm{mWh} / \mathrm{m}^{2}\right)\end{array}$ & $\begin{array}{l}\text { PAR Mittelwert } \\
\left(\mathrm{mmol} / \mathrm{m}^{2} \mathrm{~s}\right)\end{array}$ & $\begin{array}{l}\text { UVB Mittelwert } \\
\left(\mathrm{mWh} / \mathrm{m}^{2}\right)\end{array}$ \\
\hline Periode 1 & $30.08 .-11.09 .2018$ & 696 & 7968 & 54 & 613 \\
Periode 2 & $12.09 .-24.09 .2018$ & 602 & 6627 & 46 & 510 \\
Periode 3 & $25.09 .-03.10 .2018$ & 370 & 4054 & 41 & 450 \\
Periode 4 & $04.10 .-19.10 .2018$ & 598 & 6046 & 37 & 378 \\
Gesamt & $30.08 .-19.10 .2018$ & 2266 & 24.695 & 44 & 484 \\
\hline
\end{tabular}

Nur in diesem Bereich verbesserten weder die (recycelte) Aluminiumfolie noch die Textilfolie Lumilys in jeder als auch in jeder 2. Reihe die Rotfärbung.

Dagegen fielen die hue-Farbwinkel in Bodennähe unter $1 \mathrm{~m}$ Höhe auf der Ostseite bei den Früchten mit Stimplex (66, $4^{\circ}$ hue), aus der Kontrolle mit Gras (69, $1^{\circ}$ hue) und nach Folienwechsel in P. 2 (70,4 ${ }^{\circ}$ hue) ganz besonders hoch aus. Diese schlechtere Rotfärbung im unteren Kronenbereich ermöglichte den Reflexionsfolien ihre Wirkung am stärksten zu entfalten - mit guter Rotfärbung der Apfelfrüchte direkt über der Aluminiumfolie (53,3hue) und über der Textilfolie Lumilys in jeder (59,6 hue) als auch jeder 2. Reihel Fahrgasse (57hue).

Das Auslegen von Lumilys jede 2. Reihe zeigte in allen 4 betrachteten Bereichen (Quadranten des Baumes) fast identische hue-Farbwinkel wie beim herkömmlichen Auslegen in jeder Reihe. Bei den beiden Varianten, bei denen Lumilys mehrfach $(3 \times)$ in derselben Reihe verschoben wurde, waren die Apfelfrüchte bei dem Zyklus des Wechsels P. 1 besser gefärbt, was sich mit geringeren hue-Farbwinkel im Vergleich zu den hohen hue-Farbwinkeln von Wechsel P. 2 zeigte. Die Apfelfrüchte bei Wechsel P. 1 wiesen in einer Höhe über $1 \mathrm{~m}$ auf der Westseite mit $50^{\circ}$ hue den kleinsten Wert auf und somit die beste Rotfärbung (Abb. 4).

Die Apfelfrüchte, unter denen die recycelte Aluminiumfolie ausgelegt wurde, wiesen in allen vier Bereichen des Baumes die gleichen hue-Farbwinkel auf. Apfelfrüchte unter $1 \mathrm{~m}$ Baumhöhe hatten sowohl mit 53,3 ${ }^{\circ}$ hue im Osten als auch mit $52,7^{\circ}$ hue im Westen geringe hue-Farbwinkel und somit eine gute Rotfärbung und unterschieden sich nicht von den Werten über $1 \mathrm{~m}$ Höhe mit 53,5 hue im Osten und $52,6^{\circ}$ hue im Westen. Die Äpfel über recycelter Aluminiumfolie wiesen im Bereich über $1 \mathrm{~m}$ Höhe vergleichbare Ergebnisse bzw. Rotfärbung auf wie Lumilys und übertrafen im Bereich unter $1 \mathrm{~m}$ Höhe die Rotfärbung mit Lumilys (Abb. 4).

\section{Wetterdaten}

Die Summen an UVB- und photosynthetisch aktiver Strahlung sowie der Mittelwert pro Tag für die 4 Messzeiträume (Tab. 2) entsprechen den Zeiträumen, in denen die Foli- en bei dem Folienwechsel Parzelle 1+2 lagen (Abb. 3). An den Mittelwerten pro Tag erkennt man sowohl bei der UVB so wie auch bei der PAR-Strahlung die Abnahme des Lichtes im Herbst. Der mittlere UVB Wert in der ersten Periode sank von $613 \mathrm{mWh} / \mathrm{m}^{2}$ auf $378 \mathrm{mWh} / \mathrm{m}^{2}$ in der letzten Messperiode. Bei den Mittelwerten von PAR verhält es sich ähnlich, hier sank der Wert von der ersten Periode von $54 \mathrm{mmol} / \mathrm{m}^{2} \mathrm{~s}$ auf $37 \mathrm{mmol} / \mathrm{m}^{2} \mathrm{~s}$. Die erste Messperiode zeichnete sich auch durch die höchste Summe an UVBund photosynthetisch aktiver Strahlung aus. Der geringste Wert wurde jeweils in der 3. Periode festgestellt. Der zweit höchste Wert befindet sich in der 2. Periode und der dritt größte nicht in der dritten, sondern in der vierten Messperiode, welche mit 16 Tagen wesentlich länger war als die anderen drei (Tab. 2).

In den Zeiträumen, in denen die Lumilys Folie (dreimal) verschoben wurde (Wechsel P.1 und Wechsel P.2 Abb. 2) war sowohl die PAR- als auch die UVB-Strahlung wesentlich stärker als die Folie an der Ostseite von P.1 und der Westseite von P.2 lag (Abb. 5). Die Apfelbäume in P.1 erhielten $\mathrm{zu}$ der Zeit mit Lumilys auf der Ostseite mit $602 \mathrm{mmol} \mathrm{PAR} / \mathrm{m}^{2} \mathrm{~s}$ und $7228 \mathrm{mWh} / \mathrm{m}^{2}$ fast doppelt so viel Licht (PAR; UVB) als zu der Zeit, als die Folie auf der Westseite lag (336 mmol PAR/m² s und $\left.3638 \mathrm{mWh} / \mathrm{m}^{2}\right)$.

\section{Diskussion}

\section{Beurteilung der klimatischen Bedingungen für die Rotfärbung der Apfelschale}

Für die Rotfärbung der Apfelschale ist die Aktivität der drei Gene MdMYB1, MdMYB10 und bHLH3 von Bedeutung. Die Induktion der Anthocyanbiosynthese erfolgt zu Beginn der Fruchtreife (MYB10) über diese drei Gene, MYB1 reagiert auf Licht (PAR) und MYB10 auf kühle Temperaturen bzw. großen Temperaturunterschied zwischen Tag und Nacht (Takos et al. 2006; Treutter 2010). Eine ausreichende Lichtmenge steigert die Genexpression von MYB1 und bHLH3. Diese interagieren mit MYB1 und MYB10 Genen und nehmen so Einfluss auf die Biosynthese von Anthocyan (Abb. 6). Ihre Transkription wird durch kältere Tem- 
Abb. 5 Summe an PAR und UVB-Strahlung für die Ostund Westseite mit und ohne Lumilys für die Parzellen 1 und 2 bei 3 maligem Verschieben der Lumilys Folie
Summe (PAR;UVB)

Parzelle $1 \quad$ Parzelle 2
Summe (PAR;UVB)

Parzelle $1 \quad$ Parzelle 2

\begin{tabular}{c|c}
$\begin{array}{c}\text { Lumilys }(336 \\
\mathrm{mmol} / \mathrm{m}^{2} \mathrm{~s} ;\end{array}$ & $\mathrm{Gras}(336$ \\
$\left.3638 \mathrm{mWh} / \mathrm{m}^{2}\right)$ & $\mathrm{mWh} / \mathrm{m}^{2} \mathrm{~s} ; 3638$ \\
\hline $\mathrm{Gras}(336$ & Lumilys $(336$ \\
$\mathrm{mmol} / \mathrm{m}^{2} \mathrm{~s} ; 3638$ & $\mathrm{mmol} / \mathrm{m}^{2} \mathrm{~s} ; 3638$ \\
$\left.\mathrm{mWh} / \mathrm{m}^{2}\right)$ & $\left.\mathrm{mWh} / \mathrm{m}^{2}\right)$
\end{tabular}

\begin{tabular}{c|c} 
Gras $(602$ & Lumilys $(602$ \\
$\mathrm{mmol} / \mathrm{m}^{2} \mathrm{~s} ; 7228$ & $\mathrm{mmol} / \mathrm{m}^{2} \mathrm{~s} ; 7228$ \\
$\left.\mathrm{mWh} / \mathrm{m}^{2}\right)$ & $\left.\mathrm{mWh} / \mathrm{m}^{2}\right)$ \\
\hline Lumilys $(602$ & $\mathrm{Gras}(602$ \\
$\mathrm{mmol} / \mathrm{m}^{2} \mathrm{~s} ; 7228$ & $\mathrm{mmol} / \mathrm{m}^{2} \mathrm{~s} ; 7228$ \\
$\left.\mathrm{mWh} / \mathrm{m}^{2}\right)$ & $\left.\mathrm{mWh} / \mathrm{m}^{2}\right)$
\end{tabular}

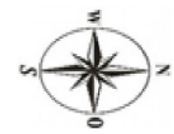

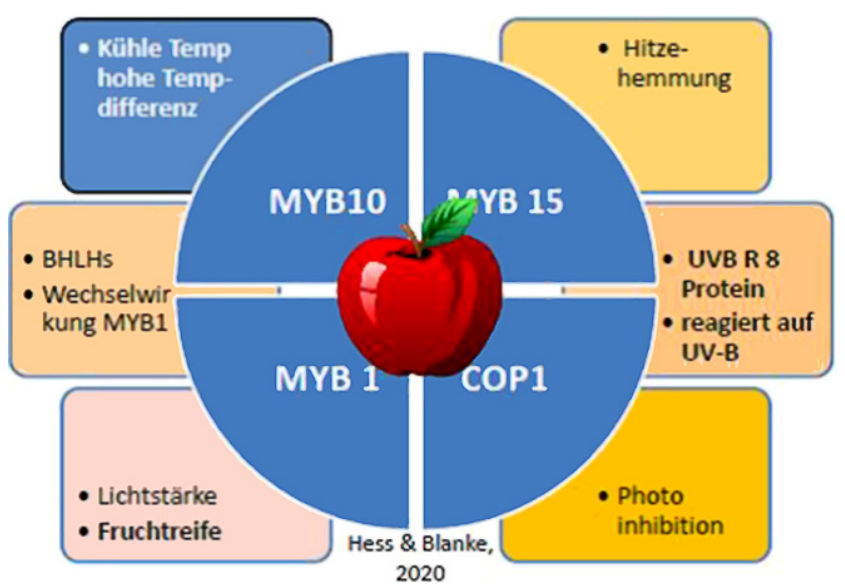

Abb. 6 Molekulargenetische Regulierung der Rotfärbung der Frucht mit den farbfördernden Genen auf der linken und hemmenden Genen bzw. Transkriptionsfaktoren auf der rechten Seite (@) M. Blanke

peraturen gefördert. Genau wie die Transkripte von MYB1 korrelieren sie positiv mit der Anthocyansynthese in der Fruchtschale. Die Interaktion von bHLH3 und MYB1 Proteinen wirkt sich also positiv auf die Anthocyanbiosynthese aus (Xie et al. 2012).

Die in Tab. 2 dargestellten Werte für PAR zeigen, dass Licht (PAR) im Herbst 2018 für eine sehr gute Rotfärbung der Apfelschale in ausreichender Menge verfügbar war. Für den Standort Klein-Altendorf bei dem, wie bei allen nördlichen Anbaugebieten, Licht der begrenzende Faktor bei der Rotfärbung der Apfelschale darstellt (Blanke 2015), kann man im Anbaujahr 2018 in Klein-Altendorf von ausreichender Lichtstärke von $2266 \mathrm{mmol} / \mathrm{m}^{2} \mathrm{~s}$ in den 6 Wochen vor der Ernte ausgehen (Tab. 2).

Die moderaten Temperaturen im September 2018 (Abb. 5) lassen vermuten, dass das Gen MYB15 nicht aktiv wurde und die Rotfärbung durch hohe Temperaturen - wie in südlichen Anbaugebieten im Spätsommer - hemmen konnte. Die Wechselwirkung zwischen Licht und Temperatur zeigt sich darin, dass die Transkripte des hitzeempfindlichen MYB1 die Transkripte des lichtabhängigen MYB1 hem- men. Dasselbe wie für PAR gilt auch für die UVB-Strahlung, die über das Protein UVR8 die Rotfärbung fördert. Durch die in Tab. 2 dargestellten Summen und Mittelwerte für UVB-Strahlung lässt sich eine durch das Protein UVR8 bewirkte Stressreaktion in der Apfelschale auf die UVBStrahlung schlussfolgern (Rizzini et al. 2011; Christie et al. 2012; Wu et al. 2012). Die ausreichende Menge an PAR lässt ebenfalls eine geringe Aktivität des Gens COP1 folgern. Die Transkripte von COP1 hemmen die Transkripte von MYB1 (Mellway et al. 2009). Im Anbaujahr 2018 in Klein-Altendorf stand eine ausreichende Menge an PAR und UVB-Strahlung im Bestand für eine überdurchschnittliche Rotfärbung der Apfelschalen der Sorte 'Braeburn, Hillwell` und anderer Sorten zur Verfügung.

\section{Einfluss der Position am Baum von den Äpfeln auf ihre Rotfärbung}

Die hue-Farbwinkel der Äpfel auf der Ostseite lagen generell höher, d.h. die Früchte waren schlechter gefärbt als auf der Westseite der Baumreihen. Bei einer Süd-Nord Ausrichtung der Baumreihen wie in Klein-Altendorf nach den Empfehlungen von Jackson und Palmer (1972) steht die Sonne nachmittags im Westen. Die photosynthetisch aktive Strahlung sowie die UVB-Strahlung weisen zu den Mittagsstunden und nachmittags höhere Werte auf als am Vormittag, an dem die Sonne im Osten steht. Die bessere Rotfärbung der Äpfel auf der Westseite lässt sich mit dem höheren Niveau an photosynthetisch aktiver Strahlung sowie UVB-Strahlung am Nachmittag erklären. Durch das höhere Strahlungsniveau gibt es vermutlich auf der Westseite eine höhere Expression des Gens Md MYB1. Das Gen MYB15 dürfte bei diesem Punkt keine bedeutende Rolle spielen. Es ist zwar davon auszugehen, dass auf der Westseite aufgrund der höheren Strahlung auch höhere Temperaturen zu bemessen sind (Solomakhin und Blanke 2008), jedoch würden diese die Rotfärbung behindern und nicht fördern (Treutter 2010). 
Apfelfrüchte auf der schlecht belichteten Ostseite unter $1 \mathrm{~m}$ zeigten bei der einfaktoriellen Varianzanalyse signifikante Unterschiede in der Rotfärbung zwischen Reflexionsfolien und Kontrolle. Bisher war nicht bekannt, auf welcher Baumseite und in welcher Baumhöhe Reflexionsfolien am stärksten/besten wirken (z.B. Schuhknecht et al. 2018).

\section{Beurteilung der Wirkung der Reflexionsfolie Lumilys, der Aluminiumfolie sowie von Stimplex}

Das Biostimulanz Stimplex zeigte keine signifikante Wirkung auf die Rotfärbung der Apfelfrüchte im Vergleich zur unbehandelten Kontrolle. Die gerichtete Reflexion der PAR und UVB-Strahlung (Meinhold et al. 2010a) durch die Aluminiumfolie färbte die Apfelfrüchte besonders gut und gleichmäßig in allen vier Quadranten. Besonders deutlich zeigt sich die Wirkung auf der Ostseite unter $1 \mathrm{~m}$ Höhe und ebenfalls auf der Westseite unter $1 \mathrm{~m}$ Höhe. Die Apfelfrüchte hatten hier im Vergleich zur Kontrolle mit Gras oder auch im Vergleich zu den Varianten mit Lumilys eine bessere Rotfärbung. Lumilys bewirkt eine diffuse Reflexion des Lichtes (Meinhold et al. 2010a), welche das Licht in einem breiten Winkel reflektiert. Das beidseitige Auslegen von Lumilys und das Auslegen in jeder 2. Reihe verbesserte die Rotfärbung der Apfelfrüchte unter $1 \mathrm{~m}$ Höhe im Osten. Die bessere Wirkung der Aluminiumfolie unter $1 \mathrm{~m}$ Höhe kommt durch die größere Menge an reflektiertem Licht in die untere Baumregion zustande (Meinhold et al. 2010b). Die gerichtete Reflexion reflektiert in diesem Bereich das Licht stärker als die diffuse Reflexion von Lumilys.

Beim 3maligen Verschieben der Reflexionsfolie Lumilys in der Reihe sollten anhand zweier Parzellen die Wirkung der Lumilys Folie zu verschiedenen Zeitpunkten festgestellt werden. Die Farbmessungen haben gezeigt, dass die Äpfel aus Parzelle 1 durch die Sequenz der Wechsel besser gefärbt waren als die aus Parzelle 2 (Abb. 4). Das Vorhandensein der Folie Lumilys an der Ostseite der Baumreihe leistete im frühen Zeitraum vom 14. bis zum 21. September einen maßgeblichen Beitrag mit positiven Auswirkungen für die Rotfärbung der Äpfel in Parzelle 1. Für das sehr gute Ergebnis der Äpfel in Parzelle 1 auf der Westseite unter $1 \mathrm{~m}$ Höhe $\left(50^{\circ}\right.$ hue) scheint das Vorhandensein der Lumilys Folie in dem Zeitraum vom 25.09. bis zum 04.10.2018 auf der Westseite verantwortlich zu sein. Das bedeutet, dass sowohl die PAR- als auch die UVB-Strahlung höher war, als die Lumilys Folie auf der Ostseite von Wechsel P.1 und der Westseite von Wechsel P.2 lag. Höhere PAR und UVBStrahlung bedeutet, dass Lumilys stärker reflektieren konnte und somit die Rotfärbung der Apfelfrüchte verbessern konnte. Das Ergebnis ist eine bessere Rotfärbung der Äpfel in Parzelle 1 im Vergleich zu den Äpfeln in Parzelle 2.

Die ausschließlich auf der schwächer belichteten Ostseite unter $1 \mathrm{~m}$ Höhe nachweisbare positive Wirkung der Refle- xionsfolien sowie die Summen an PAR und UVB-Strahlung (Tab. 2) in den 6 Wochen vor der Ernte lassen den Schluss zu, dass im Erntejahr 2018 am Versuchsort Klein-Altendorf eine geeignete Kombination aus kühler Temperatur bzw. großer Temperaturdifferenz und ausreichend hohe Mengen an PAR und UVB-Strahlung zur Verfügung standen, mit Ausnahme des genannten Quadranten. Ein weiterer Beleg für diese Aussage ist die gleichmäßige Rotfärbung der Äpfel in allen 7 Varianten in einer Höhe über $1 \mathrm{~m}$ und auf der Westseite. Es ist davon auszugehen, dass bei schlechteren klimatischen Bedingungen die Wirkung der Reflexionsfolien stärker ausfallen würde.

Danksagung Die Autoren bedanken sich bei den Herstellern für Proben ihrer Produkte, bei Achim Kunz für Abb. 2 und professionelle Hilfestellung bei den Farbmessungen sowie bei HJ Wiesel für professionelle Pflege der Obstanlagen in Klein-Altendorf.

Funding Open Access funding enabled and organized by Projekt DEAL.

Interessenkonflikt P. Hess und M. Blanke geben an, dass kein Interessenkonflikt besteht.

Open Access Dieser Artikel wird unter der Creative Commons Namensnennung 4.0 International Lizenz veröffentlicht, welche die Nutzung, Vervielfältigung, Bearbeitung, Verbreitung und Wiedergabe in jeglichem Medium und Format erlaubt, sofern Sie den/die ursprünglichen Autor(en) und die Quelle ordnungsgemäß nennen, einen Link zur Creative Commons Lizenz beifügen und angeben, ob Änderungen vorgenommen wurden.

Die in diesem Artikel enthaltenen Bilder und sonstiges Drittmaterial unterliegen ebenfalls der genannten Creative Commons Lizenz, sofern sich aus der Abbildungslegende nichts anderes ergibt. Sofern das betreffende Material nicht unter der genannten Creative Commons Lizenz steht und die betreffende Handlung nicht nach gesetzlichen Vorschriften erlaubt ist, ist für die oben aufgeführten Weiterverwendungen des Materials die Einwilligung des jeweiligen Rechteinhabers einzuholen.

Weitere Details zur Lizenz entnehmen Sie bitte der Lizenzinformation auf http://creativecommons.org/licenses/by/4.0/deed.de.

\section{Literatur}

Blanke M (2015) Möglichkeiten zur Verbesserung der Rotfärbung bei Äpfeln. Erwerbs-Obstbau 57:47-62. https://doi.org/10.1007/ s10341-015-0234-X

Blanke M, Kunz A (2009) Einfluss rezenter Klimaveränderungen auf die Phänologie bei Kernobst am Standort Klein-Altendorf - anhand 50-jähriger Aufzeichnungen. Erwerbs-Obstbau 51:101-114. https://doi.org/10.1007/s10341-009-0086-3

Bosančić B, Mićić N, Blanke M, Pecina M (2018) A main effects meta principal components analysis of netting effects on fruit: using apple as a model crop. Plant Growth Regul 86(3):455-464. https:// doi.org/10.1007/s10725-018-0443-Z

Christie JM, Arvai AS, Baxter KJ, Heilmann M, Pratt AJ, O'Hara A, Kelly SM, Hothorn M, Smith BO, Hitomi K, Jenkins GI, Getzoff ED (2012) Plant UVR8 photoreceptor senses UV-B by tryptophan-mediated disruption of cross-dimer salt bridges. Science 335:1451-1452

Funke K, Blanke M (2021) Spatial and Temporal Enhancement of Colour Development in Apples Subjected to Reflective Material in the Southern Hemisphere. Horticulturae 7(1):2 
Hamadziripi E, Muller M, Theron KI, Steyn WJ (2014) Consumer preference for apple eating quality and taste in relation to canopy position. Acta Hortic 1058:253

Jackson JE, Palmer J (1972) Interception of light by model hedgerow orchards in relation to latitude, time of year and hedgerow configuration and orientation. J Appl Ecol 9:341-357

Lumilys (2018) http://www.lumilys.com/. Zugegriffen: 10. Dez. 2018

McGuire RG (1992) Reporting of objective color measurements. HortScience 27(12):1254-1255

Meinhold T, Richters J-P, Damerow L, Blanke M (2010a) Optical properties of reflection ground covers with potential for enhancing fruit colouration. Biosyst Eng 107:155-160. https://doi.org/10. 1016/j.biosystemseng.2010.07.006

Meinhold T, Damerow L, Kunz A, Blanke M (2010b) Verschiedene Materialien zur Lichtreflexion in Apfelanlagen unter Hagelnetzen zur Verbesserung der Fruchtqualität und besonders der Fruchtfarbe bei ,Gala'. Erwerbs-Obstbau 53:1-10. https://doi.org/10.1007/ s10341-010-0122-3

Meinhold T, Damerow L, Blanke M (2011) Reflective materials under hailnet improve orchard light utilization, fruit quality and particularly fruit colouration. Sci Hortic 127:447-451. https://doi.org/ 10.1016/j.scienta.2010.09.006

Mellway RD, Tran LT, Prouse MB, Campbell MM, Constabel CP (2009) The wound-, pathogen-, and ultraviolet B-responsive MYB134 gene encodes an R2R3 MYB transcription factor that regulates proanthocyanidin synthesis in poplar. Plant Physiol 150:924-941

Rizzini L, Favory JJ, Cloix C, Faggionato D, O’Hara A, Kaiserli E, Baumeister R, Schafer E, Nagy F, Jenkins GI, Ulm R (2011) Perception of UV-B by the arabidopsis UVR8 protein. Science 332:103-106

Schuhknecht H, Damerow L, Kunz A, Blanke M (2018) Einfluss von Biostimulanzien und Lichtreflexionsfolie auf die Fruchtqualität und Farbentwicklung bei Apfel. Erwerbs-Obstbau 60:89-103. https://doi.org/10.1007/s10341-017-0353-7

Solomakhin A, Blanke M (2008) Coloured hailnets alter light transmission, spectra and phytochrome, as well as vegetative growth, leaf chlorophyll and photosynthesis and reduce flower induction of apple. Plant Growth Regul 56:211-218. https://doi.org/10.1007/ s10725-008-9302-7

Steyn WJ, Wand SJ, Jacobs G, Rosecrance RC, Roberts SC (2009) Evidence for a photoprotective function of low-temperature-induced anthocyanin accumulation in apple and pear peel. Physiol Plant 136:461-472

Takos A, Jaffé F, Jacob S, Bogs J, Robinson S, Walker A (2006) Lightinduced expression of a MYB gene regulates anthocyanin biosynthesis in red apples. Plant Physiol. https://doi.org/10.1104/pp.106. 088104

Treutter D (2010) Managing phenol contents in crop plants by phytochemical farming and breeding - visions and constraints. Int $\mathbf{J}$ Mol Sci 11:807-857

Weber S, Damerow L, Kunz A, Blanke M (2019) Anthocyanin synthesis and light utilisation can be enhanced by reflective mulch visualization of light penetration into a tree canopy. J Plant Physiol 233:52-57. https://doi.org/10.1016/j.jplph.2018.12.008

Wu D, Hu Q, Yan Z, Chen W, Yan C, Huang X, Zhang J, Yang P, Deng H, Wang J, Deng XW, Shi Y (2012) Structural basis of ultraviolet-B perception by UVR8. Nature. https://doi.org/10. 1038/nature10931

Xie XB, Li S, Zhang RF, Zhao J, Chen YC, Zhao Q (2012) The bHLH transcription factor MdbHLH3 promotes anthocyanin accumulation and fruit colouration in response to low temperature in apples. Plant Cell Environ 35:1884-1897

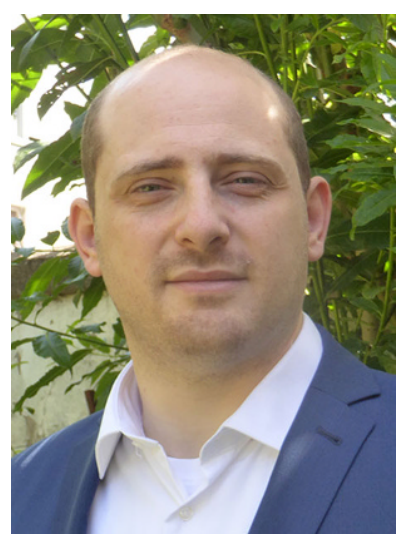

Patrick Hess studierte seit 2009 Agrarwissenschaften an der Universität Bonn und schloss sein Studium im Mai 2019 mit dem Master (zum Thema Reflexionsfolien zur Verbesserung der Fruchtqualität bei Apfel unter Hagelnetz) ab; sein besonderes Interesse gilt dem Obstbau und besonders der Verringerung des Einsatzes von Kunststoffen in der Landwirtschaft bzw. im Gartenbau. Seinen beruflichen Einstieg nach dem Master hat er beim Landwirtschaftsministerium in BonnDuisdorf gestartet. 